The final publication is available at www.degruyter.com.

DOI: https://doi.org/10.1515/ipem.2011.054. 


\section{Plasma visfatin and adiponectin concentrations in physically active adolescent girls: relationships with insulin sensitivity and body composition variables}

\author{
Jaak Jürimäe ${ }^{1}$, Rita Gruodytè ${ }^{1,2}$, Meeli Saar ${ }^{1}$, Antonio \\ Cicchella ${ }^{3}$, Claudio Stefanelli ${ }^{4}$, Catherine Passariello ${ }^{4}$, \\ Katre Maasalu ${ }^{1}$, Toivo Jürimäe ${ }^{1}$ and Serge $P$. von \\ Duvillard ${ }^{5, *}$ \\ ${ }^{1}$ Faculty of Exercise and Sport Sciences, Institute of Sport \\ Pedagogy and Coaching Sciences, Center for Behavioral \\ and Health Sciences, University of Tartu, Tartu, Estonia \\ ${ }^{2}$ Department of Physical Education and Gymnastics, \\ Lithuanian Academy of Physical Education, Kaunas, \\ Lithuania \\ ${ }^{3}$ Department of Psychology, Faculty of Exercise and Sport \\ Sciences, University of Bologna, Bologna, Italy \\ ${ }^{4}$ Department of Biochemistry "G. Moruzzi”, Faculty \\ of Exercise and Sport Sciences, University of Bologna, \\ Bologna, Italy \\ ${ }^{5}$ Department of Kinesiology/Exercise Science and \\ Biology, The College of Idaho, Caldwell, ID, USA
}

\begin{abstract}
The purpose of this study was to evaluate the associations of visfatin and adiponectin concentrations with insulin resistance and body composition in regularly physically active pubertal girls. In 129 girls, aged 13-15 years (pubertal stages 3-5), visfatin, adiponectin, insulin resistance measured by homeostasis model assessment (HOMA), and body composition measured by dual-energy X-ray absorptiometry were evaluated. Visfatin concentration was related to HOMA and overall adiposity (body mass index, fat mass) markers, whereas adiponectin concentration was related to overall adiposity (fat mass), central adiposity (trunk fat) and fat free mass values. These relationships remained significant $(\mathrm{p}<0.05)$ after adjusting for pubertal stage. Visfatin was independently related to body mass index $(\beta=0.936 ; p=0.0001)$ and HOMA $(\beta=0.444 ; \mathrm{p}=0.039)$ indices, whereas adiponectin was independently related to fat free mass $(\beta=0.889$; $\mathrm{p}=0.003)$ and trunk fat $(\beta=-0.468 ; \mathrm{p}=0.042)$ values. In conclusion, visfatin could be related to insulin resistance and overall adiposity indices, whereas adiponectin was related to different body composition values in regularly physically active pubertal girls.
\end{abstract}

*Corresponding author: Serge P. von Duvillard, PhD, FACSM, FECSS, Department of Kinesiology/Exercise Science and Biology, College of Idaho, 2112 Cleveland Blvd., Caldwell, ID 83605, USA Phone: +1-208-459-5830, Fax: +1-208-459-5854,

E-mail: svonduvillard@collegeofidaho.edu
Keywords: adipokines; female adolescents; insulin resistance; visceral obesity.

\section{Introduction}

Adipose tissue is not merely a site of energy storage but also the largest endocrine organ that releases numerous adipokines. Vast evidence suggests that adipokines secreted from adipose tissue, such as leptin, tumor necrosis factor- $\alpha$ (TNF $\alpha$ ), interleukin-6 (IL-6), and resistin modulate insulin sensitivity and appear to play an important role in the development of the metabolic syndrome $(1,2)$. The production, release, and plasma concentrations of these adipose tissue-derived hormones have been investigated extensively in healthy normal weight $(3,4)$, obese $(1,5)$, and also physically active $(6,7)$ children and adolescents. Excess adiposity, the most common cause of insulin resistance in children $(3,8)$, can influence the association between these adipokines and insulin resistance $(5,7)$. In addition, lower levels of adiponectin are related to greater insulin resistance in children and adolescents $(3,9,10)$. Adiponectin is one of the most abundant circulating adipose tissue-specific adipokines, which is mainly produced by adipose tissue (11). Because adiponectin appears to respond to acute and chronic exercise $(7,12)$, regular physical activity can modify the association between adiponectin and insulin resistance. Adiponectin has been reported to be higher in physically active adolescent females compared to sedentary controls (13).

Visfatin, also known as pre-B cell colony-enhancing factor, is an adipokine that is also highly expressed in visceral adipose tissue (14). It has also been suggested that visfatin serves as a link between visceral obesity and type 2 diabetes mellitus $(15,16)$ as visceral obesity is known to be a risk factor for the development of insulin resistance and type 2 diabetes mellitus $(14,17)$. However, other investigators have found no difference in the expression of visfatin in visceral and subcutaneous adipose tissue $(18,19)$. Furthermore, Varma et al. (20) revealed that visfatin is highly expressed in subcutaneous adipose tissue in relatively lean and more insulin-sensitive subjects. Previous studies of visfatin in children and adolescents have provided somewhat conflicting results. A significant positive association between plasma visfatin and body mass index (BMI) has been suggested in some studies (17), whereas others have reported no correlation between visfatin and indices of overall adiposity $(1,3,15)$. In addition, Fernandez-Real et al. (21) reported an independent association between visfatin concentration 
and insulin resistance in healthy lean adults, whereas previous studies in children reported no independent relationship between visfatin and insulin resistance in physically inactive obese children and adolescents $(1,3,15)$. However, given that insulin sensitivity has been reported to improve with chronic exercise training (22), it could be postulated that visfatin could also be involved in the regulation of insulin sensitivity in regularly physically active adolescents. The present study was designed to evaluate the association of plasma visfatin concentration with insulin sensitivity and body composition variables in regularly physically active pubertal girls. In addition, we also examined the relationship between plasma adiponectin, insulin sensitivity, and body composition.

\section{Methods}

A total of 129 healthy pubertal girls, aged 13-15 years, participated in this study. The volunteers were recruited from local training groups and had a training history in their selected sports for the past 2 years (6). Pubertal girls had been training for the past $4.6 \pm 2.0$ years with a mean training volume of $6.7 \pm 3.5 \mathrm{~h} /$ week (Table 1). Studied girls participated in various sports such as basketball $(\mathrm{n}=18)$, volleyball $(\mathrm{n}=15)$, badminton $(\mathrm{n}=14)$, track and field $(\mathrm{n}=22)$, gymnastics $(n=22)$, swimming $(n=23)$, and cross-country skiing $(n=15)$. None of the participants took medications or had a history of endocrine, metabolic, or kidney diseases. Throughout the study, no restrictions were placed on dietary intake and all subjects consumed their ordinary everyday diet (6). The study was approved by the Medical Ethics Committee of the University of Tartu in Estonia. The purposes, risks, and benefits were explained to the girls and their parents who signed the consent forms.

All testing was completed during the two visits of the subjects to the laboratory. During the first visit, participants had a fasting $10-\mathrm{mL}$ venous blood sample collected. In addition, biological age and main anthropometric parameters were assessed. The first measurement session was conducted during the early follicular phase of the menstrual cycle in menstruating girls $(6,23)$. The second measurement session consisted of body composition assessment by dual-energy

Table 1 Subject characteristics $(n=129)$.

\begin{tabular}{lll}
\hline Variables & Mean $( \pm \mathrm{SD})$ & Range \\
\hline Age, years & $14.0 \pm 1.0$ & $13-15$ \\
Pubertal stage $(1 / 2 / 3 / 4 / 5)$ & $4(3-5)$ & $0 / 0 / 19 / 92 / 18$ \\
Years of training & $4.6 \pm 2.1$ & $2.0-8.0$ \\
Training duration, h/week & $6.7 \pm 3.5$ & $3.0-16.0$ \\
Height, cm & $165.5 \pm 7.3$ & $149.1-186.8$ \\
Body mass, kg & $54.8 \pm 8.9$ & $38.2-80.2$ \\
BMI, kg/m ${ }^{2}$ & $20.0 \pm 2.6$ & $16.8-24.6$ \\
$\%$ FM & $24.5 \pm 6.9$ & $11.6-42.7$ \\
FM, kg & $13.2 \pm 5.5$ & $4.7-21.9$ \\
Trunk fat, kg & $5.8 \pm 2.8$ & $1.7-10.5$ \\
FFM, kg & $41.4 \pm 5.3$ & $30.5-58.3$ \\
Glucose, mmol/L & $4.9 \pm 0.4$ & $3.7-6.1$ \\
Insulin, $\mu I U / m L$ & $6.3 \pm 4.1$ & $2.0-23.3$ \\
HOMA score & $1.37 \pm 0.92$ & $0.36-4.66$ \\
Adiponectin, $\mu \mathrm{g} / \mathrm{mL}$ & $14.6 \pm 6.6$ & $0.89-33.85$ \\
Visfatin, $\mathrm{ng} / \mathrm{mL}$ & $0.9 \pm 0.8$ & $0.2-4.6$ \\
\hline
\end{tabular}

X-ray absorptiometry (DXA). Measurement sessions were separated by approximately 1 week, dependent on the participant's schedule and DXA availability.

Pubertal development of the participants was assessed based on self-assessment using an illustrated questionnaire of pubertal stages according to the Tanner classification method (24). Pubertal development assessment according to the Tanner method, which uses self-assessment of breasts and pubic hair stage in girls, has been validated previously (25) and used in our previous studies with adolescent female subjects (6). The girls were given photographs, figures and descriptions, and asked to choose the one that most accurately reflected their appearance. In case of discrepancies between the two variables (breast development and pubic hair stage), greater emphasis for the determination of Tanner stage was placed on the degree of breast development $(6,26)$.

Body height was measured using a Martin metal anthropometer to the nearest $0.1 \mathrm{~cm}$ with a standardized technique. Body mass was measured with minimal clothing to the nearest $0.05 \mathrm{~kg}$ using a medical electronic scale (A\&D Instruments, Abingdon, UK), and BMI was calculated as body mass $(\mathrm{kg})$ divided by height $\left(\mathrm{m}^{2}\right)$. Whole-body fat mass (FM) and fat free mass (FFM) were measured via DXA using the DPX-IQ densitometry (Lunar, Madison, WI, USA) equipped with proprietary software, version 3.6. Participants were scanned in light clothing while lying supine with their arms at their sides. The standard participant positioning was used for total body measurements and analyzed using the extended analysis option. The standard manufacturer's skeletal landmarks were used to define trunk fat. Trunk fat was used as a marker of central adiposity (27).

A 10-mL blood sample was obtained from an antecubital vein with the participant sitting in an upright position in the morning $(07.00$ $08.00 \mathrm{~h}$ ) after an overnight fast. The plasma was separated and frozen at $-20^{\circ} \mathrm{C}$ for later analysis. Blood plasma analysis was completed within 6 months after collection. Visfatin and total adiponectin were measured with enzyme-linked immunosorbent assay (ELISA) kits (AdipoGen and Mediagnost, Aspenhaustr, Germany). The intra- and inter-assay coefficient of variation (CV) for visfatin was $<10 \%$ and $8 \%$, and for total adiponectin was $<5 \%$ and $6 \%$, respectively. Insulin was analyzed on an Immulite 2000 (DPC, Los Angeles, CA, USA). The intra- and inter-assay CV was $<5 \%$ and $12 \%$, respectively, at an insulin concentration of $6.6 \mu \mathrm{IU} / \mathrm{mL}$. Glucose was measured using the hexokinase/glucose-6-phosphate-dehydrogenase method with a commercial kit (Böhringer, Mannheim, Germany). The insulin resistance index from fasting plasma insulin and plasma glucose levels was estimated using the homeostasis model assessment (HOMA), as follows: fasting plasma insulin $(\mu \mathrm{IU} / \mathrm{mL}) \times$ fasting plasma glucose $(\mathrm{mmol} / \mathrm{L}) / 22.5$ (28). The greater the HOMA value, the greater was the level of insulin resistance.

Statistical analysis was performed using SPSS (SPSS Inc., Chicago, IL, USA) 15.0 for Windows, and means and standard deviations $( \pm \mathrm{SD})$ were calculated. Evaluation of the normality was performed with the Shapiro-Wilk statistical test. Log transformation was performed for non-normally distributed variables. Relationships of plasma visfatin and adiponectin concentrations with other measured variables were assessed by Pearson product moment correlation and partial correlation coefficient analyses. Multiple linear regression analyses were performed using plasma visfatin and adiponectin concentrations as the dependent variables and using pubertal stage, years of training, BMI, FM, trunk fat, FFM, insulin, and HOMA as independent variables. Significance was set at $\mathrm{p}<0.05$. A sample size power calculation indicated that 129 participants were sufficient to perform the study with a power of $80 \%$ and an $\alpha$ error of $5 \%$. 


\section{Results}

The mean $( \pm \mathrm{SD})$, minimum, and maximum values of measured characteristics for the study population are presented in Table 1. Most of the studied participants were at pubertal stage $4(n=92)$, whereas 19 were at pubertal stage 3 , and 18 were at pubertal stages 5 , respectively.

Plasma visfatin concentration was significantly related $(\mathrm{p}<0.05)$ to BMI ( $r=0.187$; Figure $1 \mathrm{~A}), \mathrm{FM}(\mathrm{r}=0.149)$, and HOMA ( $\mathrm{r}=0.165$; Figure 1B) values. Partial correlation analyses revealed that plasma visfatin concentration was still related $(\mathrm{p}<0.05)$ to BMI $(\mathrm{r}=0.184), \mathrm{FM}(\mathrm{r}=0.154)$ and HOMA $(\mathrm{r}=0.158)$ values after controlling for pubertal stage. There was an inverse association $(\mathrm{p}<0.05)$ of plasma adiponectin concentration with body mass $(r=-0.170), F M(r=-0.153)$, and trunk fat $(\mathrm{r}=-0.168$; Figure $2 \mathrm{~A})$ values in adolescent female athletes. In addition, a positive association $(\mathrm{p}<0.05)$ between adiponectin and FFM was observed ( $\mathrm{r}=0.161$; Figure $2 \mathrm{~B})$. These relationships remained significant $(\mathrm{p}<0.05)$ after adjustment for pubertal status for body mass ( $\mathrm{r}=-0.175)$, trunk fat $(\mathrm{r}=-0.155)$, and FFM $(\mathrm{r}=0.151)$ indices but not for FM $(r=-0.115 ; \mathrm{p}>0.05)$ value.

Using multiple linear regression analysis, visfatin was related to BMI $(\beta=0.936 ; p=0.0001)$ and HOMA $(\beta=0.444$; $\mathrm{p}=0.039$ ) indices, whereas adiponectin was related to FFM $(\beta=0.889 ; \mathrm{p}=0.003)$ and trunk fat $(\beta=-0.468 ; \mathrm{p}=0.042)$ values.

\section{Discussion}

In the present study, we investigated whether plasma visfatin and adiponectin concentrations are related to specific insulin sensitivity and body composition parameters in regularly physically active pubertal girls. The results of our investigation indicated that plasma visfatin concentration was positively related to insulin resistance (HOMA) and overall adiposity (BMI, FM) markers, whereas plasma adiponectin
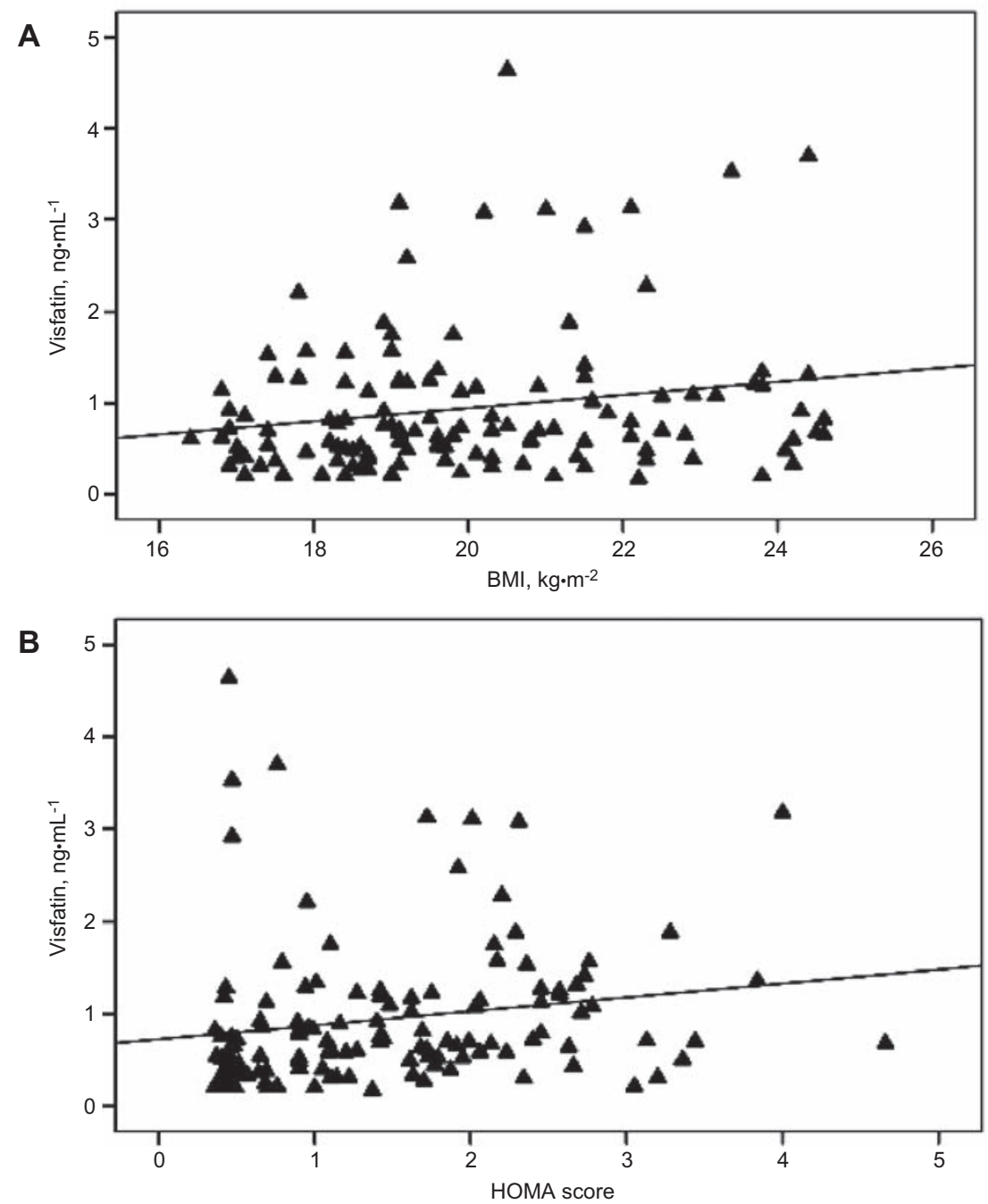

Figure 1 Relationship of plasma visfatin concentration to body mass index (BMI) (A) (r=0.19; p<0.05) and HOMA score (B) (r=0.17; $\mathrm{p}<0.05)$. 

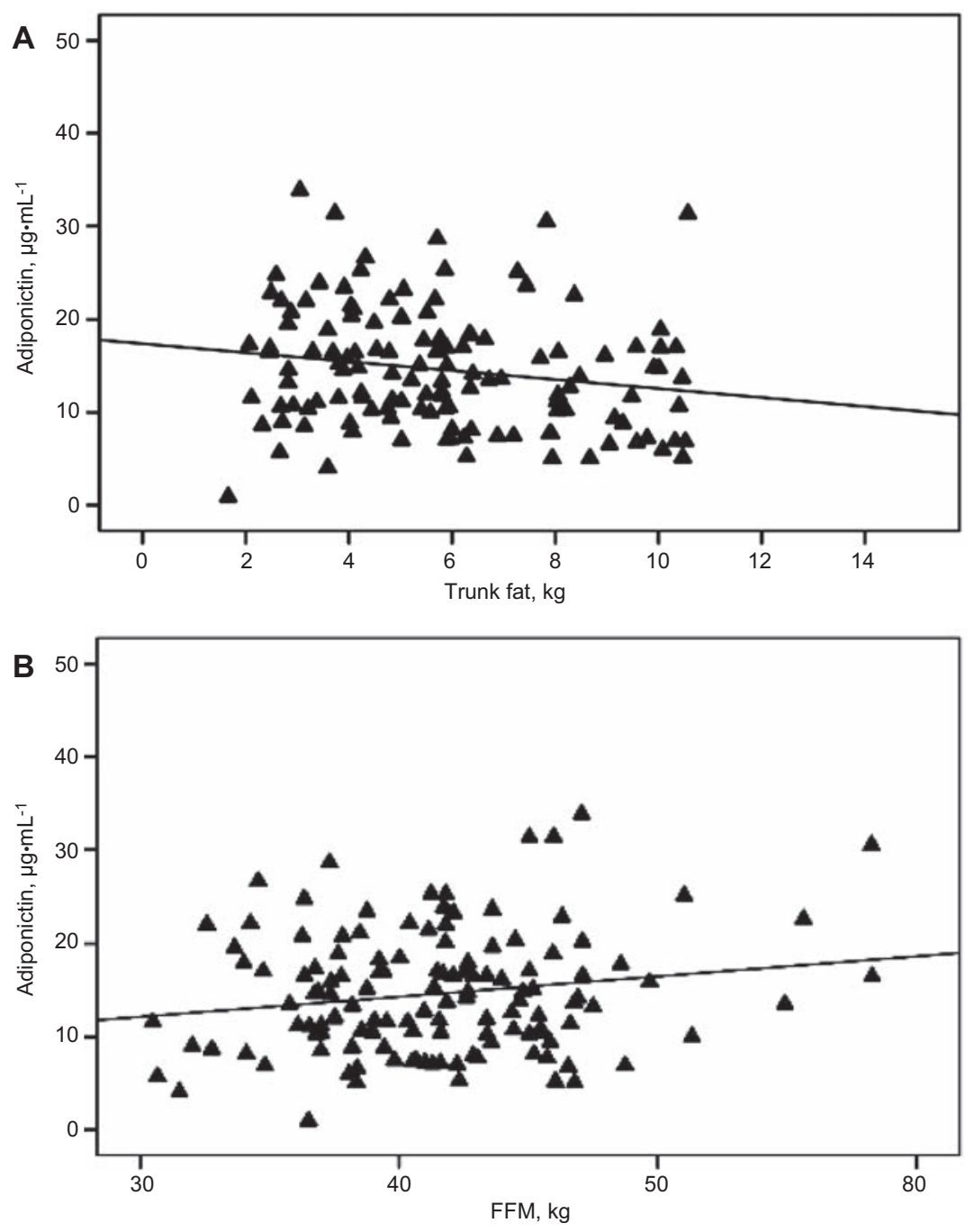

Figure 2 Relationship of plasma adiponectin concentration with trunk fat $(\mathrm{A})(\mathrm{r}=-0.17 ; \mathrm{p}<0.05)$ and fat free mass $(\mathrm{FFM})(\mathrm{B})(\mathrm{r}=0.16$; $\mathrm{p}<0.05)$.

concentration was negatively related to overall (FM) and central (trunk fat) adiposity values, and positively related to FFM in regularly physically active adolescent females. To our knowledge, this could be the first report to describe the plasma visfatin concentrations in regularly physically active pubertal girls. Plasma visfatin levels in these girls were similar and lower than the visfatin concentrations obtained in physically inactive normal weight and overweight children of similar age (15). The adiponectin concentrations in the present study were similar to the adiponectin concentrations reported in previous studies with regularly physically active female adolescents $(13,29)$. In addition, adiponectin concentrations have been reported to be higher in physically active adolescents in comparison to sedentary adolescents $(13,29)$.

The main finding of the present investigation was that an independent association between circulating visfatin concentration and insulin resistance was observed in regularly physically active adolescent females. To date, the only published studies with visfatin and insulin resistance in children also found that visfatin concentrations correlated with insulin and HOMA in heterogeneous groups of obese children with both sexes and various pubertal stages $(1,17)$. However, a positive relationship between visfatin and HOMA disappeared in these children when multivariate analysis was performed $(1,17)$. In support of these findings, Fernandez-Real et al. (21) also reported an independent association between visfatin concentration and insulin resistance in healthy nonobese subjects. However, other studies have found that circulating visfatin levels were not related to insulin resistance as assessed by HOMA in different groups of adults with a wide range of ages and adiposity values $(19,30,31)$. Choi et al. (30) reported that 12 weeks of exercise training program causing a significant decrease in body mass values was accompanied by a significant decrease in visfatin and HOMA scores in both normal weight and obese women. By contrast, given that insulin sensitivity has been reported to improve with exercise training $(22,30)$, the positive association between plasma visfatin and insulin resistance in the 
regularly physically active adolescent girls studied here suggests that visfatin could be involved in the regulation of insulin sensitivity and a decreased visfatin concentration could exert a positive effect on insulin sensitivity in regularly physically active pubertal girls. Although the role of the visfatin gene in lipid metabolism has been suggested, the underlying mechanism is currently unknown (32). In addition, some studies have reported correlations between visfatin and highdensity lipoprotein cholesterol only in obese adolescents and adults $(3,15,33)$. Accordingly, different adiposity and physical activity levels could have a different contribution to circulating visfatin concentration. However, further studies are needed to ascertain reliable conclusions.

It is clear that adipose tissue is not simply a reservoir for excess nutrients but an active endocrine organ which releases various factors into the circulation. There is increasing evidence of the association between insulin resistance and inflammation involving different cytokines $(14,31)$. The importance of these cytokines that influence metabolic parameters has recently expanded (14) and the literature increasingly implicates these adipose tissue-derived factors as major regulators of insulin sensitivity (2). Adiponectin has been reported to be associated with the indices of insulin resistance in overweight children $(3,9,10)$ and adults $(22,27,34)$. It has been suggested that the protective role of adiponectin in insulin resistance is more relevant in overweight adolescents, whereas the protective role of adiponectin is minimal in normal weight adolescents (35). This could at least partially explain why visfatin and not adiponectin in the present study was independently related to insulin resistance in regularly physically active pubertal girls $(\beta=0.444$; $\mathrm{p}=0.039$ ). The results of the present study indicate that circulating visfatin could have exerted an effect on insulin sensitivity in our specific group of regularly physically active adolescent females.

Positive correlations of plasma visfatin concentrations with indices of overall adiposity (BMI, FM) were found in this study $(r>0.149 ; \mathrm{p}<0.05)$. In addition, BMI was an independent variable $(\beta=0.936 ; p=0.0001)$ characterizing plasma visfatin levels in physically active adolescent females. The association between visfatin and BMI is in accordance with other studies in children (17) and adults $(18,30)$. By contrast, it has been found that visfatin concentration is not associated with BMI in normal weight $(1,3)$ and obese $(3,15)$ children. Likewise, other studies have reported no correlation between visfatin and overall adiposity values in adults with a wide range of adiposity values $(14,21,36,37)$. Furthermore, Araki et al. (1) suggested that plasma visfatin is a surrogate marker for visceral fat accumulation in obese children. However, other investigations did not find any difference in the expression of visfatin between subcutaneous and visceral adipose tissues $(18,19)$. A possible reason for the conflicting results of our study and other studies $(1,3,15)$ could be the fact that our subjects were older and more developed in their pubertal stage with relatively lean body composition values and higher physical activity levels. In addition, discrepancies in laboratory measurement methods could also account for these differences $(3,17)$. In accordance with a study by Berndt et al. (18), the results of the present investigation suggest that circulating plasma visfatin is associated with overall adiposity values. The reason that no association was found between visfatin concentration and trunk fat could be due to the relatively low level of trunk fat in the physically active study subjects. However, further interventional studies are necessary to evaluate the relationship of plasma visfatin to different adiposity values.

In addition to the association between circulating adiponectin and overall adiposity (FM) $(38,39)$ in physically active adolescent females, measured central adiposity level (trunk fat) might also have contributed to plasma adiponectin concentration as reported in other studies $(9,39)$. The relationships were similar for measured overall and central adiposity indices in regularly physically active adolescent females, whereas other studies have indicated that central fat distribution is a better determinant of circulating adiponectin than total FM in heterogeneous groups of adolescent children (9). An interesting finding of the present study was that an independent association of plasma adiponectin concentration with FFM value ( $\beta=0.889 ; p=0.003$ ) was observed in the studied pubertal girls. These results imply that in addition to body adipose tissue values, other body composition factors such as FFM could modulate the plasma adiponectin levels. This is consistent with the results of our previous study of middleaged premenopausal women, where an independent association between adiponectin and FFM values was observed in women who had experienced significant alterations in energy homeostasis but not in women who had relatively stable body mass values (27). Indeed, the participants in the present study were physically active girls with increased energy expenditure values. However, further studies are needed before any conclusions can be drawn.

The strength of this study lies in the fact that the study population was healthy and regularly physically active adolescent girls who have already reached puberty. To the best of our knowledge, this relatively homogeneous group of subjects has not been studied previously. However, we recognize that the selection of this group is not representative of the general population of the same age and pubertal development. In addition, the cross-sectional nature of our study limits determinations of temporality or causality. Therefore, further interventional studies are necessary to evaluate the relationship of visfatin and adiponectin with different insulin resistance and body composition values. Another limitation was that the use of a more sensitive measure of insulin resistance than HOMA could strengthen the results of the present study.

In conclusion, these data support the hypothesis that circulating visfatin could be linked to insulin resistance and overall adiposity indices, whereas adiponectin concentration is related to different body composition values in a group of regularly physically active adolescent females.

\section{Acknowledgment}

This study was supported by Estonian Science Foundation Grant No. 8086. 


\section{Conflict of interest statement}

The authors declare that they have no conflict of interest. Experimental procedures were performed in accordance to the Declaration of Helsinki and comply with the current laws of Estonia.

\section{References}

1. Araki S, Dobashi K, Kubo K, Kawagoe R, Yamamoto Y, et al. Plasma visfatin concentration as a surrogate marker for visceral fat accumulation in obese children. Obesity 2008;16:384-8.

2. Sharma AM, Chetty VT. Obesity, hypertension and insulin resistance. Acta Diabetol 2005;42(Suppl 1):S3-8.

3. Jin H, Jiang B, Tang J, Lu W, Wang W, et al. Serum visfatin concentrations in obese adolescents and its correlation with age and high-density lipoprotein cholesterol. Diabetes Res Clin Pract 2008;79:412-8.

4. Pomerants T, Tillmann V, Karelson K, Jürimäe J, Jürimäe T. Ghrelin response to acute aerobic exercise in boys at different stages of puberty. Horm Metab Res 2006;38:752-7.

5. Moon Y, Kim D, Song D. Serum tumor necrosis factor-alpha levels and components of the metabolic syndrome in obese adolescents. Metabolism 2004;53:863-7.

6. Jürimäe J, Cicchella A, Jürimäe T, Lätt E, Haljaste K, et al. Regular physical activity influences plasma ghrelin concentration in adolescent girls. Med Sci Sports Exerc 2007;39:1736-41.

7. Nemet D, Wang P, Funahashi T, Matsuzawa Y, Tanaka S, et al. Adipocytokines, body composition, and fitness in children. Pediatr Res 2003;53:148-52.

8. Caprio S. Insulin resistance in childhood obesity. J Pediatr Endocrinol Metab 2002;15(Suppl 1):487-92.

9. Huang KC, Lue BH, Yen RF, Shen CG, Ho SR, et al. Plasma adiponectin levels and metabolic factors in nondiabetic adolescents. Obes Res 2004;12:119-24.

10. Pilz S, Horejsi R, Moller R, Almer G, Scharnagl H, et al. Early atherosclerosis in obese juveniles is associated with low serum levels of adiponectin. J Clin Endocrinol Metab 2005;90:4792-6.

11. Motoshima H, Wu X, Sinha MK, Hardy VE, Rosato EL, et al. Differential regulation of adiponectin secretion from cultured human omental and subcutaneous adipocytes: effects of insulin and rosiglitazone. J Clin Endocrinol Metab 2002;87:5662-7.

12. Jürimäe J, Purge $P$, Jürimäe T. Adiponectin and stress hormone responses to maximal sculling after volume-extended training season in elite rowers. Metabolism 2006;55:13-9.

13. Ischander M, Zaldivar F Jr, Eliakim A, Nussbaum E, Dunton $\mathrm{G}$, et al. Physical activity, growth, and inflammatory mediators in BMI-matched female adolescents. Med Sci Sports Exerc 2007;39:1131-8.

14. Chen MP, Chung FM, Chang DM, Tsai JCR, Huang HF, et al. Elevated plasma level of visfatin/pre-B cell colony-enhancing factor in patients with type 2 diabetes mellitus. J Clin Endocrinol Metab 2006;91:295-9.

15. Haider DG, Holzer G, Schaller G, Weghuber D, Widhalm K, et al. The adipokine visfatin is markedly elevated in obese children. J Pediatr Gastroenterol Nutr 2006;43:548-9.

16. Sethi JK, Vidal-Puig A. Visfatin: the missing link between intra-abdominal obesity and diabetes? Trends Mol Med 2005; 11:344-7.

17. Davutoglu M, Ozkaya M, Guler E, Garipardic M, Gursoy H, et al. Plasma visfatin concentration in childhood obesity: relationships to insulin resistance and anthropometric indices. Swiss Med Wkly 2009;139:22-7.
18. Berndt J, Kloting N, Kralisch S, Kovacs P, Fasshauer M, et al. Plasma visfatin concentrations and fat depot-specific mRNA expression in humans. Diabetes 2005;54:2911-6.

19. Pagano C, Pilon C, Olivieri M, Mason P, Fabris R, et al. Reduced plasma visfatin/pre-B cell colony-enhancing factor in obesity is not related to insulin resistance in humans. J Clin Endocrinol Metab 2006;91:3165-70.

20. Varma V, Yao-Borengasser A, Rasouli N, Bodles AM, Phanavanh $B$, et al. Human visfatin expression: relationship to insulin sensitivity, intramyocellular lipids, and inflammation. J Clin Endocrinol Metab 2007;92:666-72.

21. Fernandez-Real JM, Moreno JM, Chico B, Lopez-Bermejo A, Ricart W. Circulating visfatin is associated with parameters of iron metabolism in subjects with altered glucose tolerance. Diabetes Care 2007;30:616-21.

22. Hulver MW, Zheng D, Tanner CJ, Houmard JA, Kraus WE, et al. Adiponectin is not altered with exercise training despite enhanced insulin action. Am J Physiol Endocrinol Metab 2002;283:E861-5.

23. Thong FS, McLean C, Graham TE. Plasma leptin in female athletes: relationship with body fat, reproductive, nutritional, and endocrine factors. J Appl Physiol 2000;88:2037-44.

24. Tanner J. Growth at adolescence, 2nd ed. Oxford, UK: Blackwell Scientific Publications, 1962.

25. Matsudo SMM, Matsudo VKR. Self assessment and physician assessment of sexual maturation in Brazilian boys and girls: concordance and reproducibility. Am J Hum Biol 1994;6: $451-5$.

26. Lehtonen-Veromaa M, Möttönen T, Irjala K, Nuotio I, Leino A, et al. 1-year prospective study on the relationship between physical activity, markers of bone metabolism, and bone acquisition in peripubertal girls. J Clin Endocrinol Metab 2000;85:3726-32.

27. Jürimäe $J$, Jürimäe T, Ring-Dimitriou S, LeMura LM, Arciero $\mathrm{PJ}$, et al. Plasma adiponectin and insulin sensitivity in overweight and normal-weight middle-aged premenopausal women. Metabolism 2009;58:638-43.

28. Matthews DR, Hosker JP, Rudenski AS, Naylor BA, Treacher DF, et al. Homeostasis model assessment: insulin resistance and beta-cell function from fasting plasma glucose and insulin concentrations in men. Diabetologia 1985;28:412-9.

29. Rubin DA, McMurray RG, Harrell JS, Thorpe DE, Hackney AC. Vigorous physical activity and cytokines in adolescents. Eur J Appl Physiol 2008;103:495-500.

30. Choi KM, Kim JH, Cho GJ, Baik SH, Park HS, et al. Effect of exercise training on plasma visfatin and eotoxin levels. Eur J Endocrinol 2007; 157:437-42.

31. Oki K, Yamane K, Kamei N, Nojima H, Kohno N. Circulating visfatin level is correlated with inflammation, but not with insulin resistance. Clin Endocrinol 2007;67:796-800.

32. Jian WX, Luo TH, Gu YY, Zhang HL, Zheng S, et al. The visfatin gene is associated with glucose and lipid metabolism in a Chinese population. Diabet Med 2006;23:967-73.

33. Chen CC, Li TC, Li CI, Liu CS, Lin WY, et al. The relationship between visfatin levels and anthropometric and metabolic parameters: association with cholesterol levels in women. Metabolism 2007;56:1216-20.

34. Jürimäe J, Rembel K, Jürimäe T, Rehand M. Adiponectin is associated with bone mineral density in perimenopausal women. Horm Metab Res 2005;37:297-302.

35. Rubin DA, McMurray RG, Harrell JS, Hackney AC, Thorpe DE, et al. The association between insulin resistance and cytokines in adolescents: the role of weight status and exercise. Metabolism 2008;57:683-90. 
36. Jürimäe J, Rämson R, Mäestu J, Purge P, Jürimäe T, et al. Plasma visfatin and ghrelin response to prolonged sculling in competitive male rowers. Med Sci Sports Exerc 2009;41:137-43.

37. Sandeep S, Velmurugan K, Deepa R, Mohan V. Serum visfatin in relation to visceral fat, obesity, and type 2 diabetes mellitus in Asian Indians. Metabolism 2007;56:565-70.

38. Gavrila A, Chan JL, Yiannakouris N, Kontogianni M, Miller LC, et al. Serum adiponectin levels are inversely associated with overall and central fat distribution but are not directly regulated by acute fasting or leptin administration in humans: crosssectional and interventional studies. J Clin Endocrinol Metab 2003;88:4823-31.

39. Jürimäe J, Jürimäe T. Plasma adiponectin concentration in healthy pre- and postmenopausal women: relationship with body composition, bone mineral, and metabolic variables. Am J Physiol Endocrinol Metab 2007;293:E42-7. 